\title{
MAGNETIC FIELD STRENGTHS OF SPIRAL GALAXIES AND THE FAR-INFRARED/RADIO CORRELATION
}

\author{
A. J. FITT and P. ALEXANDER \\ M.R.A.O., Cavendish Laboratory, \\ Madingley Road, \\ Cambridge, CB3 OHE, \\ England.
}

We have calculated equipartition magnetic fields for a complete, optically-selected sample of 165 spiral galaxies. The magnetic field distribution (fig. 1) is type independent, and shows remarkably little spread in values, around 1 decade in $B$. This is not due to selection effects because of the nature of the sample and the 95 percent detection rate.

Unlike Hummel (1986) we find no relationship between the scatter in the far-infrared/radio correlation and the variation in magnetic field strengths (fig. 2), neither can this explain the non-linear nature of the correlation. We conclude that two-temperature dust models (Fitt, Alexander and Cox, 1988) are a better explanation of the non-linearity, and that the scatter in the correlation is not primarily due to magnetic field variations between galaxies.

Fig. 1 : B distribution

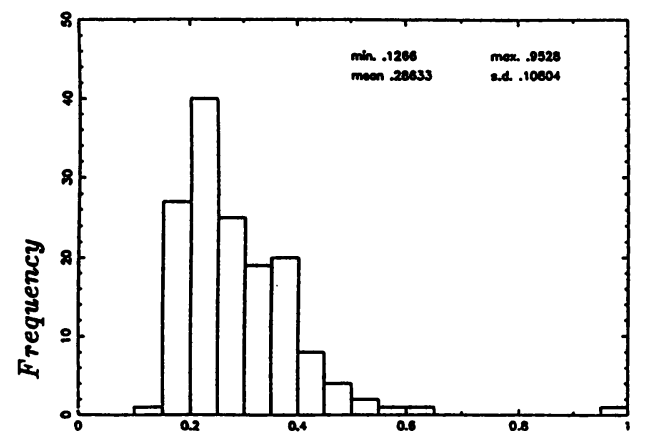

$B_{\text {equipartition }}(n T)$
Fig. 2 : Scatter in the FIR/radio correlation vs. B

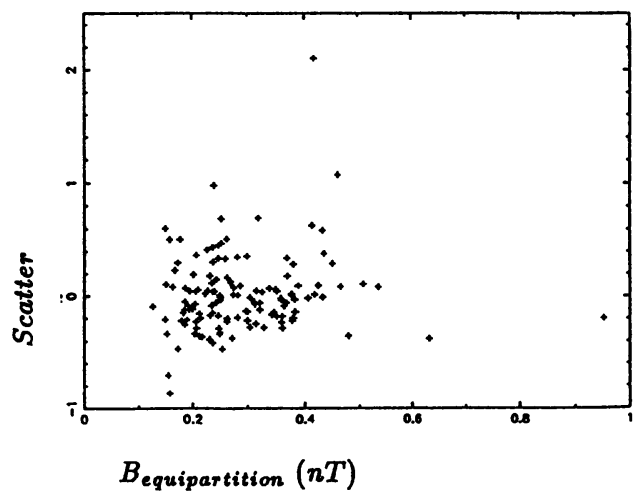

\section{References}

Fitt A.J., Alexander P. and Cox M.J., 1988. Mon. Not. R. Astr. Soc., 233, 907. Hummel, E., 1986. Astr. Astrophys., 160, L4. 